\title{
Plasma neurofilament heavy chain is not a useful biomarker in
}

\section{Charcot-Marie-Tooth Disease}

Alexander M Rossor ${ }^{1} \mathrm{PhD}$, Ching-Hua Liu ${ }^{2,4} \mathrm{PhD}$, Axel Petzold ${ }^{3} \mathrm{PhD}$, Andreas Malaspina ${ }^{4} \mathrm{PhD}$, Matilde Laura ${ }^{1} \mathrm{PhD}$, Linda Greensmith ${ }^{1} \mathrm{PhD}$, Mary M Reilly ${ }^{1} \mathrm{MD}$.

1. MRC Centre for Neuromuscular Diseases, UCL Institute of Neurology and National Hospital for Neurology and Neurosurgery, Queen Square, London, UK

2. Sobell Department of Motor Neuroscience and Movement Disorders, UCL Institute of Neurology, Queen Square, London, UK

3. VU Medical Centre, Dept. of Neurology, Amsterdam, The Netherlands

4. Centre for Neuroscience and Trauma, Blizard Institute, Barts and The School of Medicine and Dentistry, Queen Mary University of London, London, UK

\section{Acknowledgements:}

AMR has received fellowship funding from the National Institutes of Neurological Diseases and Stroke and office of Rare Diseases (U54NS065712) and an IPSEN clinical research fellowship. MMR is grateful to the Medical Research Council (MRC), MRC Centre grant (G0601943), and the National Institutes of Neurological Diseases and Stroke and office of Rare Diseases (U54NS065712) for their support. The INC (U54NS065712) is a part of the NCATS Rare Diseases Clinical Research Network (RDCRN). RDCRN is an initiative of the Office of Rare Diseases Research (ORDR), NCATS, funded through a collaboration between NCATS and the NINDS. This research was also supported by the National Institute for Health Research University College London Hospitals Biomedical Research Centre. 


\section{Corresponding author:}

Dr Alexander M Rossor

MRC Centre for Neuromuscular Diseases, 8-11 Queen Square, London, WC1N 3BG

Email: a.rossor@ucl.ac.uk 


\begin{abstract}
Background: The negative trials of vitamin C in Charcot-Marie-Tooth disease (CMT) type $1 \mathrm{~A}$ have highlighted the lack of sensitive outcome measures. Neurofilaments are abundant neuronal cytoskeletal proteins and their concentration in blood is likely to reflect axonal breakdown. We therefore examined plasma neurofilament heavy chain ( $\mathrm{NfH}$ ) concentration as a potential biomarker in CMT.
\end{abstract}

Methods: Blood samples were collected from healthy controls and patients with CMT over a 2-year period. Disease severity was measured using the CMT Examination Score. An inhouse ELISA was used to measure plasma $\mathrm{NfH}$ levels.

Results: There was no significant difference in plasma NfH concentrations between CMT patients and controls ( $P=0.449)$. There was also no significant difference in plasma $\mathrm{NfH}$ levels in the CMT group over 1 year (mean difference $=-0.02$, Standard error of the mean $=$ 4.44, $P=0.98)$.

Conclusions: Plasma NfH levels are not altered in patients with $\mathrm{CMT}$ and are not a suitable biomarker of disease activity.

Keywords:

Charcot-Marie-Tooth disease

CMT

Outcome measure

Neurofilament heavy chain

ELISA

Plasma 



\section{Introduction}

Charcot-Marie-Tooth disease (CMT) is the commonest genetic neuromuscular disease; it has a population prevalence of 1 in 2500 (1). Although no pharmacological treatments exist, there have been several successful pre-clinical trials in mouse models of the disease (2-5). Vitamin $C$ was shown to be effective in a mouse model of CMT1A (6), but this effect was not borne out in several double-blind randomized placebo-controlled trials (7-10). These trials, however, have highlighted the insensitivity of current outcome measures to detect changes in disease progression over a 2-year period and the need for a sensitive biomarker.

To date, plasma neurofilament heavy chain $(\mathrm{NfH})$ levels have been shown to be raised in several central nervous system diseases including amyotrophic lateral sclerosis (ALS) and optic neuritis (11-15). Neurofilaments are the most abundant neuronal cytoskeletal protein in peripheral nerves, and their concentration in blood likely reflects axonal breakdown and provides a biomarker of peripheral nerve disease (16). In further support of this hypothesis has been the demonstration of raised plasma $\mathrm{NfH}$ levels in a mouse model of lower motor neuron degeneration and in patients with diabetic polyneuropathy $(17,18)$.

The aims of this study were to determine whether plasma $\mathrm{NfH}$ levels are elevated in patients with CMT. If they are raised, we questioned whether they correlate with disease severity and whether they change over a 12-month follow up period.

\section{Materials and methods}

This study was approved by The National Hospital for Neurology and Neurosurgery Research Ethics Committee/ Central London REC 3 09/H0716/61. 
Blood samples were collected prospectively, with informed consent, from CMT patients attending the inherited neuropathy clinic at the NHNN. CMT1A is the commonest type of CMT and the only type of CMT in which clinical trials have taken place. Although it is classified as a demyelinating neuropathy, CMT1A patients were included in this study, as their clinical deficit is known to be due to axonal damage. Only patients with a genetically confirmed diagnosis of CMT were included, and patients with other neurological diseases were excluded. The disease severity, as measured by the Charcot-Marie-Tooth Examination Score (CMTES, second version), was recorded at the same time as plasma was collected (19). The CMTES is a validated outcome measure for assessing the severity of CMT. It is a composite score that includes the patient's symptoms and examination findings.

Blood samples were obtained from healthy relatives of patients attending the inherited neuropathy clinic and staff at the UCL Institute of Neurology. Healthy subjects were excluded if they had co-existent neurological disease as determined by a symptom- and past medical history-based questionnaire. Plasma from healthy controls was also acquired with informed consent from the relatives of patients with ALS recruited as part of a separate study [East London and the City Research Ethics Committee 1 (09/H0703/27)].

A modified in-house sandwich ELISA was used to measure plasma hyper-phosphorylated $\mathrm{NfH}$ levels in the plasma (SMI-34R, Covance, USA)(20). 
All statistical analysis was performed using SPSS version 14.00. Plasma NfH levels were compared using a Mann-Whitney $\mathrm{U}$ test, and correlation of plasma $\mathrm{NfH}$ and disease severity and age were assessed using Pearson correlation coefficients.

\section{Results}

A similar number of patients with CMT $(n=90)$ and healthy controls $(n=79)$ were included in this study. Controls were significantly older than the CMT cohort ( $P=0.039, t$-test) although the mean difference was only 5 years. There was also a significant difference in the ratio of men to women in the 2 groups [46:44 (CMT), 56:23 (Controls), $\chi^{2}=6.876 P=0.009$ ], with significantly more women in the controls. Nevertheless, there were no gender differences in plasma NfH levels within the CMT (unpaired $t$-test, $P=0.3$ ) and control groups $(P=0.9)$. The percentage of patients with demyelinating forms of $\mathrm{CMT}(71 \%)$ was higher than for axonal forms (29\%).

There was no difference in the primary outcome measure of plasma $\mathrm{NfH}$ concentration between patients with CMT $(27.4 \mathrm{ng} / \mathrm{ml})$ and controls $(21.5 \mathrm{ng} / \mathrm{ml})(P=0.449$, Mann Whitney $\mathrm{U}$ test) and no significant difference in plasma $\mathrm{NfH}$ levels between patients with axonal (27.8 $\mathrm{ng} / \mathrm{ml})$ and demyelinating $(27.3 \mathrm{ng} / \mathrm{ml})$ forms of CMT $(P=0.833)$ (see Figure 1$)$.

There was a small but significant difference in the mean age of CMT patients (44) and controls (48.9) $(P=0.02, t$-test), although there was no significant correlation between plasma $\mathrm{NfH}$ levels and age in the controls (Pearson correlation coefficient $=-0.039$, $P=0.730)$.

To determine whether the lack of a difference in plasma $\mathrm{NfH}$ levels in $\mathrm{CMT}$ is due to the inclusion of patients with a lower CMTES score $(<10)$, and hence lower levels of axonal 
degeneration, $\mathrm{NfH}$ levels were correlated against the CMTES score. Unexpectedly, there was a trend toward a reduction in $\mathrm{NfH}$ levels with increasing disease severity as defined by the CMTES (Pearson Rank -0.173, $P=0.104$ ) (Figure 1).

There was no significant difference in plasma NfH levels in the CMT group over 1 year (mean difference $=-0.02, \mathrm{SEM}=4.44$, paired $t$-test, $P=0.98)$. This is despite a mean increase in the CMTES of 1 point for all forms of CMT (SEM=0.41, $P=0.01)$.

\section{Discussion}

A cross-sectional comparison of plasma NfH levels revealed no difference between CMT patients and controls. There are several reasons why this may be the case. First, CMT is a slowly progressive disease in comparison to more rapidly progressive neurodegenerative disorders in which plasma and serum NfH levels were elevated (15). Second, the timing of clinical progression due to axonal degeneration and timing of sampling is critical, and this cohort might, while being systematic, not have covered random but clinically relevant events. The third possibility is that chronic release of $\mathrm{NfH}$ into the peripheral circulation induces an immune response that leads to the production of anti-NfH antibodies and a consequent reduction in detectable plasma $\mathrm{NfH}$ levels. This phenomenon has been observed in other neurological conditions, including primary and secondary progressive MS, in which higher antibody levels against neurofilament light chain (NfL) and $\mathrm{NfH}$ have recently been described $(21,22)$. It is also worth noting that the mean age of subjects was 44 years. It is possible that plasma NfH levels may be elevated in pediatric patients with $\mathrm{CMT}$ in whom the volume of distribution is smaller and in whom antibodies against NfH and NfL (if present) may not yet have been produced. 
Much of the work examining NfL and NfH has focused on levels in both plasma and CSF $(23,24)$. It is possible that $\mathrm{NfH}$ levels are elevated in the CSF of patients with CMT but are degraded rapidly in the plasma. Against this hypothesis is the fact that CMT is a lengthdependent, dying-back neuropathy in which neurodegeneration occurs in the distal limbs. The degree of neurodegeneration at the level of the intradural nerve root is therefore likely to be low.

In order for plasma NfH to be a biomarker of axonal degeneration in $\mathrm{CMT}$ there should be a significant difference between patients with CMT and controls, and the levels should correlate with disease severity. Unfortunately, plasma $\mathrm{NfH}$ is not higher in $\mathrm{CMT}$ patients than controls, and there is a decrease in plasma $\mathrm{NfH}$ with increasing disease severity. Plasma $\mathrm{NfH}$ levels are therefore not suitable as a biomarker of CMT disease severity or as a potential biomarker for natural history studies or clinical trials.

\author{
Abbreviations: \\ $\mathrm{CMT}=$ Charcot-Marie-Tooth disease \\ $\mathrm{NfL}=$ Neurofilament light chain \\ $\mathrm{NfH}=$ Neurofilament heavy chain \\ CMTES = Charcot-Marie-Tooth examination score \\ CMTNS = Charcot-Marie-Tooth neuropathy score \\ ALS = Amyotrophic Lateral Sclerosis \\ SEM $=$ Standard error of the mean \\ ELISA = Enzyme Linked Immuno- absorbant Assay \\ NHNN = National Hospital for Neurology and Neurosurgery
}




\section{References}

1. Skre H. Genetic and clinical aspects of Charcot-Marie-Tooth's disease. Clin Genet. $1974 ; 6: 98-118$.

2. d'Ydewalle C, Krishnan J, Chiheb DM, Van Damme P, Irobi J, Kozikowski AP, et al. HDAC6 inhibitors reverse axonal loss in a mouse model of mutant HSPB1-induced Charcot-Marie-Tooth disease. Nat Med. 2011;17:968-74.

3. Patzkó A, Bai Y, Saporta MA, Katona I, Wu X, Vizzuso D, et al. Curcumin derivatives promote Schwann cell differentiation and improve neuropathy in R98C CMT1B mice. Brain. 2012;135:3551-66.

4. Sereda MW, Meyer zu Hörste G, Suter U, Uzma N, Nave K-A. Therapeutic administration of progesterone antagonist in a model of Charcot-Marie-Tooth disease (CMT-1A). Nat Med. 2003;9:1533-7.

5. Garofalo K, Penno A, Schmidt BP, Lee H-J, Frosch MP, von Eckardstein A, et al. Oral Lserine supplementation reduces production of neurotoxic deoxysphingolipids in mice and humans with hereditary sensory autonomic neuropathy type 1. J Clin Invest. 2011;121:4735-45.

6. Passage E, Norreel JC, Noack-Fraissignes P, Sanguedolce V, Pizant J, Thirion X, et al. Ascorbic acid treatment corrects the phenotype of a mouse model of Charcot-MarieTooth disease. Nat Med. 2004;10:396-401.

7. Pareyson D, Reilly MM, Schenone A, Fabrizi GM, Cavallaro T, Santoro L, et al. Ascorbic acid in Charcot-Marie-Tooth disease type 1A (CMT-TRIAAL and CMT-TRAUK): a double-blind randomised trial. Lancet Neurol. 2011;10:320-8.

8. Burns J, Ouvrier RA, Yiu EM, Joseph PD, Kornberg AJ, Fahey MC, et al. Ascorbic acid 
for Charcot-Marie-Tooth disease type 1A in children: a randomised, double-blind, placebo-controlled, safety and efficacy trial. Lancet Neurol. 2009;8:537-44.

9. Micallef J, Attarian S, Dubourg O, Gonnaud P-M, Hogrel J-Y, Stojkovic T, et al. Effect of ascorbic acid in patients with Charcot-Marie-Tooth disease type 1A: a multicentre, randomised, double-blind, placebo-controlled trial. Lancet Neurol. 2009;8:1103-10.

10. Lewis RA, McDermott MP, Herrmann DN, Hoke A, Clawson LL, Siskind C, et al. Highdosage ascorbic acid treatment in Charcot-Marie-Tooth disease type 1A: results of a randomized, double-masked, controlled trial. JAMA Neurol. 2013;70:981-7.

11. Petzold A, Plant GT. The diagnostic and prognostic value of neurofilament heavy chain levels in immune-mediated optic neuropathies. Mult Scler Int. 2012;2012:217802.

12. Petzold A, Rejdak K, Plant GT. Axonal degeneration and inflammation in acute optic neuritis. J Neurol Neurosurg Psychiatry. 2004;75:1178-80.

13. Petzold A, Eikelenboom MJ, Keir G, Grant D, Lazeron RHC, Polman CH, et al. Axonal damage accumulates in the progressive phase of multiple sclerosis: three year follow up study. J Neurol Neurosurg Psychiatry. 2005;76:206-11.

14. Pasol J, Feuer W, Yang C, Shaw G, Kardon R, Guy J. Phosphorylated neurofilament heavy chain correlations to visual function, optical coherence tomography, and treatment. Mult Scler Int. 2010;2010:542691.

15. Boylan K, Yang C, Crook J, Overstreet K, Heckman M, Wang Y, et al. Immunoreactivity of the phosphorylated axonal neurofilament $\mathrm{H}$ subunit (pNF-H) in blood of ALS model rodents and ALS patients: evaluation of blood pNF-H as a potential ALS biomarker. J Neurochem. 2009;111:1182-91. 
16. Petzold A. Neurofilament phosphoforms: surrogate markers for axonal injury, degeneration and loss. J Neurol Sci. 2005;233:183-98.

17. Lu C-H, Petzold A, Kalmar B, Dick J, Malaspina A, Greensmith L. Plasma neurofilament heavy chain levels correlate to markers of late stage disease progression and treatment response in SOD1(G93A) mice that model ALS. PLoS One. 2012;7:e40998.

18. Qiao X, Zhang S, Zhao W, Ye H, Yang Y, Zhang Z, et al. Serum Phosphorylated Neurofilament-Heavy Chain, a Potential Biomarker, is Associated With Peripheral Neuropathy in Patients With Type 2 Diabetes. Medicine (Baltimore). 2015;94:e1908.

19. Murphy SM, Herrmann DN, McDermott MP, Scherer SS, Shy ME, Reilly MM, et al. Reliability of the CMT neuropathy score (second version) in Charcot-Marie-Tooth disease. J Peripher Nerv Syst. 2011;16:191-8.

20. Lu C-H, Kalmar B, Malaspina A, Greensmith L, Petzold A. A method to solubilise protein aggregates for immunoassay quantification which overcomes the neurofilament "hook" effect. J Neurosci Methods. 2011;195:143-50.

21. Fialová L, Bartos A, Švarcová J, Zimova D, Kotoucova J. Serum and cerebrospinal fluid heavy neurofilaments and antibodies against them in early multiple sclerosis. J Neuroimmunol. 2013;259:81-7.

22. Fialová L, Bartos A, Svarcová J, Zimova D, Kotoucova J, Malbohan I. Serum and cerebrospinal fluid light neurofilaments and antibodies against them in clinically isolated syndrome and multiple sclerosis. J Neuroimmunol. 2013;262:113-20.

23. Menke RAL, Gray E, Lu C-H, Kuhle J, Talbot K, Malaspina A, et al. CSF neurofilament light chain reflects corticospinal tract degeneration in ALS. Ann Clin Transl Neurol. 
$2015 ; 2: 748-55$.

24. Lu C-H, Petzold A, Topping J, Allen K, Macdonald-Wallis C, Clarke J, et al. Plasma neurofilament heavy chain levels and disease progression in amyotrophic lateral sclerosis: insights from a longitudinal study. J Neurol Neurosurg Psychiatry. $2015 ; 86: 565-73$. 
Figure 1. Plasma neurofilament heavy chain levels in patients with CMT versus controls. (A) the genetic breakdown of all CMT patients included in the study. (B) plasma NFH levels (black dots) of all CMT patients and controls. The horizontal dotted line shows mean plasma $\mathrm{NfH}$ levels of CMT patients $(27.4 \mathrm{ng} / \mathrm{ml})$ versus controls $(21.5 \mathrm{ng} / \mathrm{ml})(\mathrm{p}=0.449)$. (C) plasma $\mathrm{NfH}$ levels plotted against disease severity as determined by the CMTES. Unexpectedly, there was a trend toward a reduction in $\mathrm{NfH}$ levels with increasing disease severity (Pearson Rank -0.173, $P=0.104)$. (D) mean difference in plasma NfH levels over 1 year in $19 \mathrm{CMT}$ patients and 10 controls. There was no significant difference in plasma NfH levels in CMT patients (mean difference $=-0.02, P=0.98$ ) or controls (mean difference $=-7, P=0.37$ ) over 1 year. 